\title{
The Lattice Compatibility Theory: Supports from the Generalized Simha-Somcynsky Chemical Physics-Related Theory
}

\author{
K. Boubaker, ${ }^{1}$ A. Colantoni, ${ }^{2}$ and P. Petkova ${ }^{3}$ \\ ${ }^{1}$ École Supérieure de Sciences et Techniques de Tunis (ESSTT), Université de Tunis, 63 Rue Sidi Jabeur, 5100 Mahdia, Tunisia \\ ${ }^{2}$ Department of Agriculture, Forest, Nature and Energy (DAFNE), University of Tuscia, Via S. Camillo de Lellis snc, \\ 01100 Viterbo, Italy \\ ${ }^{3}$ École Shumen University "Konstantin Preslavsky", 115 Universitetska Street, 9712 Shumen, Bulgaria
}

Correspondence should be addressed to K. Boubaker; mmbb11112000@yahoo.fr

Received 16 May 2013; Accepted 12 July 2013

Academic Editor: Stefan Sokolowski

Copyright (C) 2013 K. Boubaker et al. This is an open access article distributed under the Creative Commons Attribution License, which permits unrestricted use, distribution, and reproduction in any medium, provided the original work is properly cited.

The earliest models used in the study of lattice structures are mean field theories, which do not contain structural dependence. The Lattice Compatibility Theory (LCT) proposes here a novel framework where the measure of the disorder is based on Urbach tailing features and lattice matching features between the host matrix and doping agent intrinsic structures. This study has been implemented on a particular compound (BTO:Co) and refers to the Simha-Somcynsky (SS) theory, a mean field theory where the measure of the disorder is stated as holes.

\section{Introduction}

The knowledge of doping agents behaviors within host lattice matrix is of considerable importance for the optimal design for applications such as semiconductor windows functional glasses, transparent electrodes in flat panel displays, buffer layers, and solar cells [1-9]. Although such behaviors have been studied for many host-doping agent lattice systems, theoretical fundaments and updated principles are still important for predicting or correlating the stability behavior of many systems within a wide range of lattice shapes.

The first theories based on mean field theory and independent from the design of lattice structures failed in the statistical thermodynamics of branched macromolecules. Studies on branched structures served as attempts to mathematically correct the mean field theories. One of the most important of these studies is the Lattice-Cluster Theory (LCT), developed by Freed and Bawendi [1].

For complex lattice systems, other theories have been developed. Dee and Walsh $[2,3]$ proposed the lattice theory as a tool for depicting the thermodynamic properties of heterogeneous structures. This theory was an enhancement of those of Flory $[4,5]$ and Huggins [6] concerning chain structures. Lennard-Jones and Devonshire [7,8], and Prigogine et al. [911] developed the cell model, according to which molecules can move in neighbors-induced potential holes. Later, Simha et al. $[12,13]$ noted that free volume theories, especially hole theories, could delineate the thermodynamic properties of solid structures, and they introduced the notion of free volume. The so-called Simha-Somcynsky (SS) model [12] defined accurately the holes in the lattice-like structure and determined the statistical behavior by finding out the combination of the holes formed within lattice intermolecular sites.

In the hole theory, a major change in volume is explained by the number of holes, and the change in cell size plays a minor role while in the cell theory [14-16], the changes in volume with changes in temperature and pressure can be explained only by the changes in cell size. Oppositely, the lattice theory stipulates that lattice size is fixed, and volume change is explained solely by the number of vacant sites $[15,16]$.

Zhong et al. [17] indicated that the Simha-Somcynsky (SS) model could not generate sufficient free volume and still lacks fluidity. They modified the SS model by using 


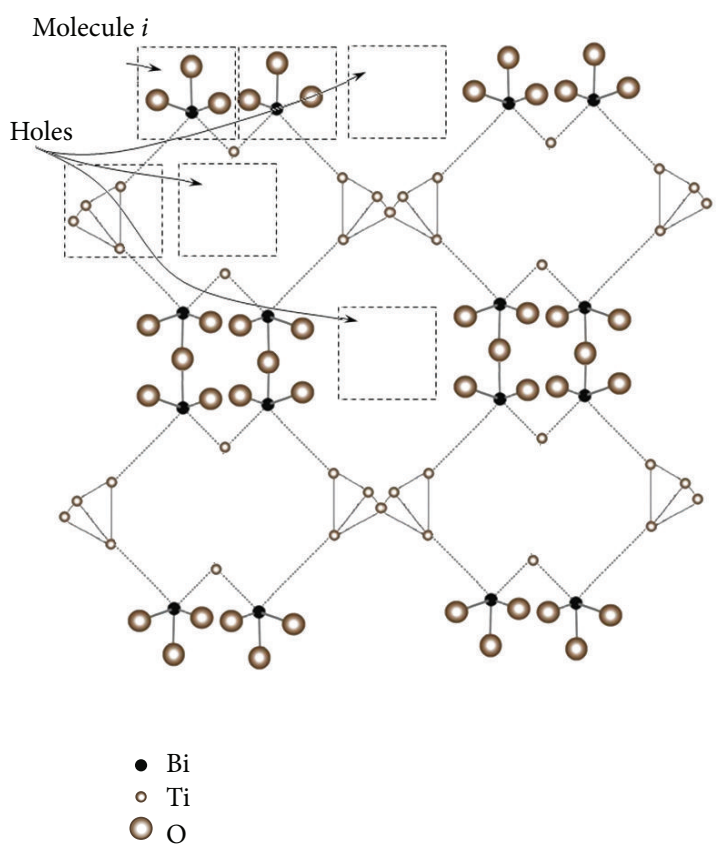

FIgURE 1: Simha-Somcynsky configuration (BTO undoped lattice).

the open-cell concept, and they induced a new empirical spatial parameter.

In this paper, it is outlined that SS theory was independent from the macromolecular architecture, and it exhibited differences in behavior concerning some doped lattices. Hence new elements for explanation of these differences are presented and discussed in the framework of the Lattice Compatibility Theory (LCT). The paper is organized in the following way. In Section 2, we present some relevant theoretical fundaments to both Simha-Somcynsky Lattice-Hole and Lattice Compatibility Theories along with some patterns of the studied BTO lattice. In Section 3, we present comparative and conjoint analyses. Section 4 is the conclusion.

\section{Theoretical Fundaments}

2.1. Elements from the Generalized Simha-Somcynsky Lattice-Hole Theory. According to the generalized Simha-Somcynsky theory $[12,13]$, any lattice can be considered as a succession of elementary molecules and holes (Figure 1). Each cell in the occupied fraction is either empty or contains the molecule van-der-Waals volume as well as an inherent free volume.

In this theory's framework, the most important aggregate which traduces entities repartition within the lattice is the volume fraction $y_{i}$. This parameter is the ratio of the occupied sites by a molecule $i$ on all of lattice sites:

$$
y_{i}=\frac{s_{i} N}{s_{i} N+N_{h}},
$$

where $N$ is the number of molecules, $N_{h}$ is the number of unoccupied cells, and $s_{i}$ is the number of equivalent segments in a molecule $i$.
The total occupied volume fraction is coupled with temperature through the minimization condition

$$
\left.\left(\frac{\partial F_{i}\left(T, y_{i}\right)}{\partial y_{i}}\right)\right|_{V, T}=0
$$

where $F_{i}\left(T, y_{i}\right)$ is the molar Helmholtz free energy for a molecule $i$ within the given structure at a temperature $T$, and approximated by

$$
\begin{aligned}
F_{i}\left(T, y_{i}\right)=R T & {\left[\ln \left(\frac{y_{i}}{s_{i}}\right)+s_{i} \frac{\left(1-y_{i}\right)}{y_{i}} \ln \left(1-y_{i}\right)\right.} \\
& \left.+\left(s_{i}-1\right)\left(1-\ln \left(z_{i}-1\right)\right)\right],
\end{aligned}
$$

where $R$ is universal gas constant $(\mathrm{J} / \mathrm{mol} \mathrm{K}), T$ is absolute temperature, and $z_{i}$ is the number of nearest neighbors of the molecule $i$.

The configurational partition function $Z_{i}\left(N, y_{i}, T\right)$ only depends on the positional part of the degrees of freedom and the potential energy of the molecule. All molecules with the same mass will have identical contributions from their kinetic degrees of freedom (the momenta), but they may have distinct modes of interacting which will result in different contributions from the configurational part. Ordinarily, the kinetic and potential energy contributions to the canonical partition function are separable. The kinetic contribution can be evaluated analytically, giving, for small molecules, the thermal de Broglie wavelength. For complex molecules, and by developing good expansion of multidimensional integral over the 3D space and statistical mechanics-related perturbation techniques, the configurational partition function is represented as

$$
Z_{i}\left(N, y_{i}, T\right)=g\left(N, y_{i}\right)\left[v_{f}\left(y_{i}\right)\right]^{c N} e^{-\left[E_{0} / k T\right]},
$$

where $g\left(N, y_{i}\right)$ is the combinatorial factor, $v_{f}$ is the free volume, $E_{0}$ is the internal energy when all of the segments are located at the center of the cell, $k$ is Boltzmann constant, and $c$ are the external degrees of freedom per molecule, as defined by Prigogine et al. [9-11] and Hirschfelder et al. [18].

The combinatorial factor $g\left(N, y_{i}\right)$, arising from the mixing of molecules and holes, represents the occupation possibilities of the molecules into the lattice sites. It is obtained through the number of molecules $N$ and volume fraction along with the number of nearest neighbors of the molecule as follows:

$$
g\left(N, y_{i}\right)=y_{i}^{-N}\left[1-y_{i}\right]^{-N\left(\left(1-y_{i}\right) / y_{i}\right)} .
$$

The free volume $v_{f}$ is, as defined by Park and Kim [19], the difference between the volume per molecule and the hardcore volume of the molecule:

$$
v_{f}=\left(\sqrt[3]{\omega}-\sqrt[3]{V^{*}} \lambda^{3}+\left[\frac{1-y_{i}}{y_{i}}\right] \sqrt[3]{\omega}\right)^{3},
$$

where $\omega$ is the cell volume, $V^{*}$ is the molecular characteristic volume, and $\lambda$ is the lattice geometric factor (i.e., $\lambda^{-2}=2$, for face-centered cubic lattice). 


\subsection{Elements from the Lattice Compatibility Theory Analysis.} The Lattice Compatibility Theory, as mentioned in some recent studies [20-23], is based on the interaction of dopingelement lattice behavior versus host edifice. Preludes to this theory have been established by Boubaker [21] in the context of analysing Urbach tailing controversial behaviour in some nanocompounds as well as I-III- $\mathrm{O}_{2}$ ternary oxide ceramics instability at low temperatures beside ternary tellurides and sulphides. They were also confirmed by Petkova and Boubaker [20] on the basis of investigation on some copperdoped bismuth sillenites (BSO) and germanates (BGO) compounds.

An original formulation of the Lattice Compatibility Theory [20-23] has been established as follows.

The stability of doping agents inside BTO host structures is favorized [sic] by geometrical compatibility, expressed in terms of matching patterns between doping agent intrinsic lattice and those of the host.

In the actual discussed case (cobalt-doped BTO lattice), the nature of the highest occupied band and the location of holes in BTO lattice structures have been demonstrated to be determinant. In this context, fundamental geometrical observations concerning the structure of BTO and the doping lattice were interpreted in terms of conventional orbitals patterns-linked geometry.

The Lattice Compatibility Theory tried to give a plausible understanding of the disparity concerning doping element incorporating dynamics starting from intrinsic doping element lattice properties in comparison to those of the host. In precedent study [20-23] materials, changes in Urbach energy have been associated with a cobalt-doping induced disorder in BTO matrices. In order to understand Urbach tailing alteration following doping agent insertion in host structures, Urbach energy $E_{u}$ has been determined for doped and undoped samples through the equations

$$
\begin{gathered}
\ln (\alpha(h \nu))=\ln \left(\alpha_{0}\right)+\frac{h \nu}{E_{u}}, \\
E_{u}=\alpha(h \nu)\left(\frac{d[\alpha(h \nu)]}{d[h \nu]}\right)^{-1}=h\left[\frac{d}{d \nu}(\ln \alpha(\nu))\right]^{-1},
\end{gathered}
$$

where $\alpha(h \nu)$ represents, for each sample, the experimentally deduced optical absorption profile.

Urbach energy $E_{u}$ is a measure of the inhomogenous disorder and atomic scale dispersion inside structures as it indicates the width of the band tails of the localized states in presence of defects. Its analytical formulation deduced takes into account three components: structural disorder, carrierphonon interaction, and carrier-impurity, as follows:

$$
\begin{aligned}
E_{u}= & \overbrace{\frac{1}{2} k_{B} U \theta_{D}}^{\text {Structural disorder }}+\overbrace{\Omega_{1} \frac{4 \pi^{2} Z^{2} q^{4} m^{*} L_{D}^{3}}{9 \sqrt{3} \varepsilon^{2} \hbar^{2}}}^{\text {Carrier-phonon interaction }} \\
& +\overbrace{\Omega_{2} \operatorname{coth}\left(\frac{\Omega_{3}}{2 k_{B} T}\right)}^{\text {Carrier-impurity }},
\end{aligned}
$$

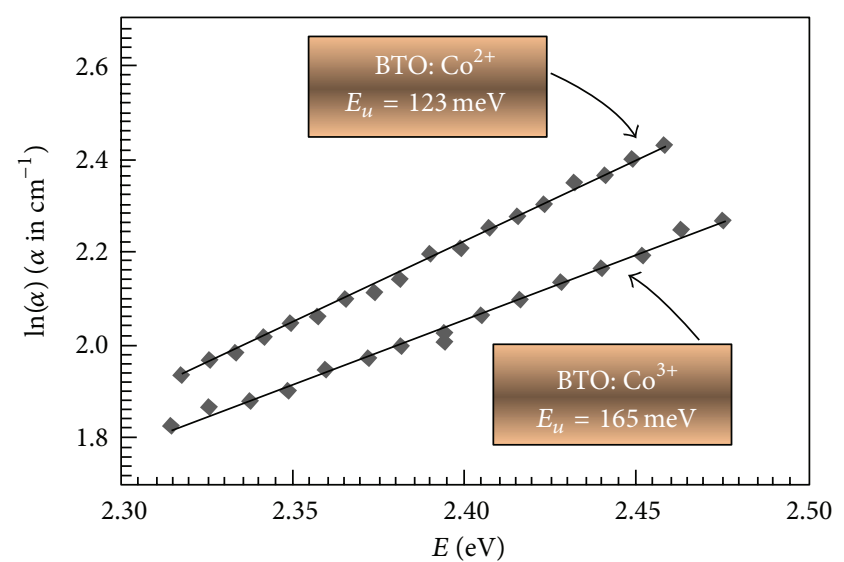

FIGURE 2: Plots of doped BTO optical coefficient versus energy (as guides to Urbach tailing quantification).

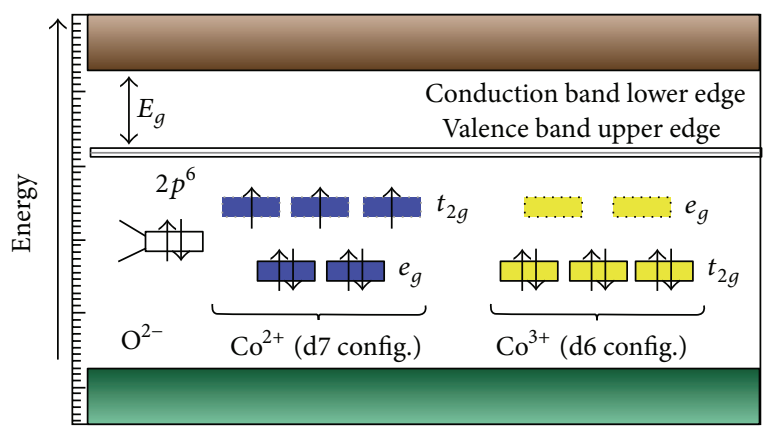

Figure 3: $\mathrm{Co}^{3+}$ and $\mathrm{Co}^{2+}$ ions outer shell configuration inside oxygen-dominant structures.

where $\Omega_{1}, \Omega_{2}$, and $\Omega_{3}$ are constants, $k_{B}$ is Boltzmann constant, $U$ is lattice strain related with the structural disorder, $\theta_{D}$ is Debye temperature, $L_{D}$ is Debye length, $m^{*}$ is carrier effective mass, $Z$ is impurity charge, $q$ is electron charge, $\varepsilon$ is static dielectric permittivity, and $\hbar$ is Planck's constant.

The width of the localized states (band tail energy or Urbach energy $E_{u}$ ) has been estimated from the slopes of the plots of $\ln \alpha(\nu)$ versus energy $h \nu$ (Figure 2). In this context, fundamental geometrical observations concerning the structure of BTO and the doping lattice are interpreted in terms of comparison to cobalt intrinsic lattice parameter (Figure 2) as well as the fact that in oxygen-rich edifices, interstitial $\mathrm{Co}^{2+}$ tetrahedral sites split the five degenerated atomic $\mathrm{d}$ orbitals into two groups, leading to 3 unpaired $\mathrm{d}$ electrons on $\mathrm{Co}^{2+}$, while all the $\mathrm{d}$ electrons of polyhedral $\mathrm{Co}^{3+}$ are paired (with a null global magnetic moment), as illustrated by Figure 3 .

Using a complete set of measurements, main lattice constants of cobalt intrinsic lattice have been compared to those of BTO and revealed an obvious compatibility with the cubic main metric and angular parameters of $\mathrm{Co}^{2+}$ intrinsic lattice. It has been recorded that $\mathrm{Co}^{2+}$ incorporation in BTO matrix was followed by a loss of the hopping motion of electrons which decreased the piling up of electrons at host matrix vacancies hence impeding the buildup of space charge 
polarization. The variation in lattice parameters with $\mathrm{Co}^{3+}$ incorporation had been attributed to the increase of the unit cell volume of the host lattice with increasing $\mathrm{Co}^{3+}$ content. In fact, since $\mathrm{Co}^{3+}$ ions are trivalent and in order to maintain the charge balance during doping, incorporation had to be accompanied by an increase of unit cell volume either by a reduction of host cations valence or an oxygen content increase.

In the same context, and according to Muncaster et al. [24], Huang et al. [25], and Chmaissem et al. [26], cobalt ions intercalation dynamics in similar host structures with fixed coordination geometries were mainly governed by differences in ionic size and magnetic properties. Relative induced disorder in BTO: $\mathrm{Co}^{3+}$ edifices show that it is fundamentally the matter of oxygen uptake. The amount of incorporated oxygen seems to be wholly dependent on the presence of additional locations where the bond lengths from oxygen to surrounding cobalt ions remain within an upper limit [26].

\section{Lattice Compatibility Theory (LCT) and Simha-Somcynsky Conjoint Analysis}

The most fundamental structure alteration which occurred in the host BTO lattice has been interpreted in terms of changes either in the configurational partition function $Z_{i}\left(N, y_{i}, T\right)$ or in the lattice parameters, according to Simha-Somcynsky analysis and Lattice Compatibility Theory, respectively.

In the Simha-Somcynsky analysis, the structure-dependent coefficients $\left(z_{i}\right.$ in (3)) increase in different ways during $\mathrm{Co}^{2+}$ and $\mathrm{Co}^{3+}$ incorporation (Figures 4 and 5).

In this context, for a molecule $i$, the relative contribution of the doping agent is expressed in terms of relative variation of the molar Helmholtz free energy as follows:

$$
\frac{\Delta F_{i}\left(T, y_{i}\right)}{\Delta z_{i}} \approx\left(s_{i}-1\right) \frac{1}{z_{i}-1}
$$

Under a first-order approximation, cobalt ions edifices presence induces a drastic decrease of Helmholtz free energy as well as a lower Urbach energy. It was recorded that these trends are not the same for $\mathrm{Co}^{2+}$ and $\mathrm{Co}^{3+}$ ions. The loss of stability which characterized the transition from $\mathrm{Co}(\mathrm{II})$ to $\mathrm{Co}(\mathrm{III})$ state and which was recorded by Amine et al. [27, 28], Duncan et al. [29], Liu et al. [30], Santhanam and Rambabu [31], and Aklalouch et al. [32,33] has been satisfactorily explained in the Lattice Compatibility Theory (LCT) framework. The most probable explanation which can be provided through the Simha-Somcynsky theory lies in the expression of the Helmholtz free energy (see (3) and (7)). Indeed, the incorporation of cobalt ions contributes to the decrease of the Helmholtz free energy [34-37], but, as it is also equivalent to the increase of the number of occupied sites within the cell (Figures 4 and 5), it induces an increase of energy through the first two terms in (3). Moreover, the latter contribution of $\mathrm{Co}^{3+}$ ions is more important due to its voluminous polyhedral configuration (Figure 5).

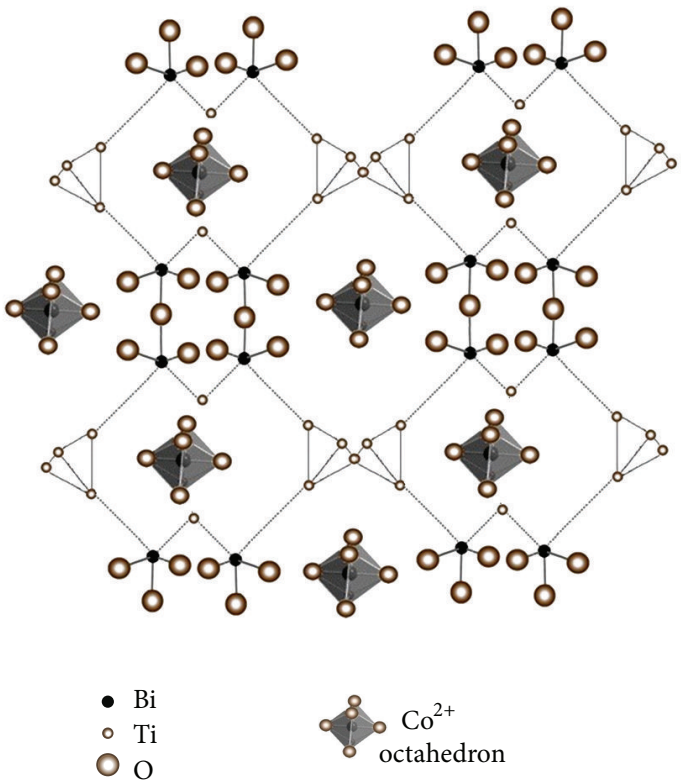

Figure 4: $\mathrm{Co}^{2+}$ ions incorporation scheme inside BTO matrix.

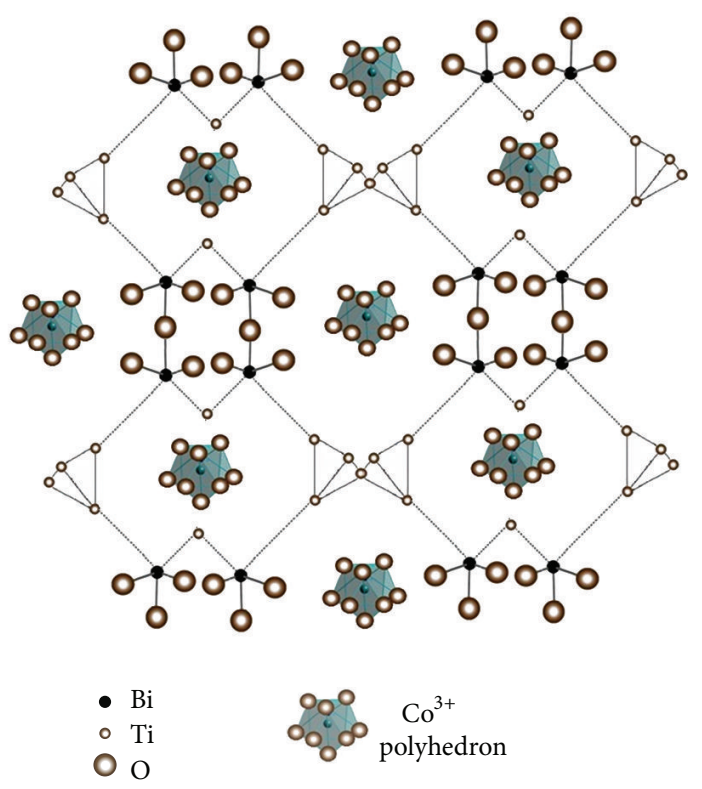

Figure 5: $\mathrm{Co}^{3+}$ ions incorporation scheme inside BTO matrix.

\section{Conclusion}

The presented work showcases some fundaments of the Lattice Compatibility Theory (LCT) framework in relation with the precedent Simha-Somcynsky theory-linked analyses. The most probable explanations which can be provided to some intriguing nanoscale unexpected alterations, through the LCT theory, have been discussed in terms of the well-known Simha-Somcynsky (SS) lattice hole theory. What this theory and more sophisticated frameworks have in common is that they incorporate a description for holes on the lattice, acting as free volumes. This approach, which became necessary since 
the lattice-based arrangement of molecules became the most encountered in the recent literature, was confronted to the results which have been recorded in an unupdated compound (BTO:Co). The results were as accurate as relevant. The LCT analyses used in lattice linear structures can also be used together with the SS model for other similar structures.

\section{Acknowledgment}

Partial financial support by project of Shumen University (2013) is gratefully acknowledged.

\section{References}

[1] K. F. Freed and M. G. Bawendi, "Lattice theories of polymeric fluids," Journal of Physical Chemistry, vol. 93, no. 6, pp. 21942203, 1989.

[2] G. T. Dee and D. J. Walsh, "Equations of state for polymer liquids," Macromolecules, vol. 21, no. 3, pp. 811-815, 1988.

[3] G. T. Dee and D. J. Walsh, "A modified cell model equation of state for polymer liquids," Macromolecules, vol. 21, no. 3, pp. 815817, 1988.

[4] P. J. Flory, "Thermodynamics of high polymer solutions," Journal of Chemical Physics, vol. 9, no. 8, pp. 660-661, 1941.

[5] P. J. Flory, "Thermodynamics of high polymer solutions," Journal of Chemical Physics, vol. 10, article 51, 1941.

[6] M. L. Huggins, "Solutions of long chain compounds," Journal of Chemical Physics, vol. 9, no. 5, p. 440, 1941.

[7] J. E. Lennard-Jones and A. F. Devonshire, "Critical phenomena in gases-II," Proceedings of the Royal Society of London A, vol. 165, pp. 1-11, 1938.

[8] J. E. Lennard-Jones and A. F. Devonshire, "Critical phenomena in gases-I," Proceedings of the Royal Society of London A, vol. 165, pp. 53-70, 1938.

[9] I. Prigogine, N. Trappeniers, and V. Mathot, The Molecular Theory of Solutions, North Holland, Amsterdam, The Netherlands, 1957.

[10] I. Prigogine, N. Trappeniers, and V. Mathot, "Statistical thermodynamics of r-MERS and r-MER solutions," Discussions of the Faraday Society, vol. 15, pp. 93-107, 1953.

[11] I. Prigogine, The Molecular Theory of Solutions, North-Holland, Amsterdam, The Netherlands, 1957.

[12] R. Simha and T. Somcynsky, "On the statistical thermodynamics of spherical and chain molecule fluids," Macromolecules, vol. 2, no. 4, pp. 342-350, 1969.

[13] P. S. Wilson and R. Simha, "Thermal expansion of amorphous polymers at atmospheric pressure-I. Experimental," Macromolecules, vol. 6, no. 6, pp. 902-908, 1973.

[14] I. C. Sanchez and R. H. Lacombe, "An elementary molecular theory of classical fluids. Pure fluids," Journal of Physical Chemistry, vol. 80, pp. 2352-2362, 1976.

[15] S. S. You, K. P. Yoo, and C. S. Lee, "An approximate nonrandom lattice theory of fluids-general derivation and application to pure fluids," Fluid Phase Equilibria, vol. 93, pp. 193-213, 1994.

[16] R. K. Jain and R. Simha, "Statistical thermodynamics of short chain molecular liquids: $n$-Nonane," Journal of Chemical Physics, vol. 70, article 2972, 1979.

[17] C. Zhong, W. Wang, and H. Lu, "Open-cell model equation of state for liquids-1. P-V-T behavior for liquids and liquid polymers," Macromolecules, vol. 27, no. 3, pp. 660-664, 1994.
[18] J. O. Hirschfelder, C. F. Curtiss, and R. B. Bird, Molecular Theory of Gases and Liquids, Wiley, New York, NY, USA, 1954.

[19] J. Park and H. Kim, "A new equation of state based on hole theory," Fluid Phase Equilibria, vol. 144, no. 1-2, pp. 77-86, 1998.

[20] P. Petkova and K. Boubaker, "The Lattice Compatibility Theory (LCT): an attempt to explain Urbach tailing patterns in copperdoped bismuth sillenites (BSO) and germanates (BGO)," Journal of Alloys and Compounds, vol. 546, no. 5, pp. 176-179, 2013.

[21] K. Boubaker, "Preludes to the Lattice Compatibility Theory LCT: urbach tailing controversial behavior in some nanocompounds," ISRN Nanomaterials, vol. 2012, Article ID 173198, 4 pages, 2012.

[22] K. Boubaker, "The lattice compatibility theory: arguments for recorded I-III- $\mathrm{O}_{2}$ ternary oxide ceramics instability at low temperatures beside ternary telluride and sulphide ceramics," Journal of Ceramics, vol. 2013, Article ID 734015, 6 pages, 2013.

[23] K. Boubaker, M. Amlouk, Y. Louartassi, and H. Labiadh, "About unexpected crystallization behaviors of some ternary oxide and sulfide ceramics within lattice compatibility theory LCT framework," Journal of the Australian Ceramic Society, vol. 49, no. 1, pp. 115-117, 2013.

[24] G. Muncaster, G. Sankar, C. R. A. Catlow, J. M. Thomas, S. J. Coles, and M. Hursthouse, "The local structure of tetrahedral $\mathrm{Co}(\mathrm{III})$ : a detailed crystal structure investigation of $\mathrm{K}_{5} \mathrm{Co}^{\mathrm{III}} \mathrm{W}_{12} \mathrm{O}_{40} \cdot 20 \mathrm{H}_{2} \mathrm{O}$," Chemistry of Materials, vol. 12 , no. 1 , pp. 16-18, 2000.

[25] Q. Z. Huang, V. L. Karen, A. Santoro et al., "Substitution of $\mathrm{Co}^{3+}$ in $\mathrm{YBa}_{2} \mathrm{Fe}_{3} \mathrm{O}_{8}$," Journal of Solid State Chemistry, vol. 172, no. 1, pp. 73-80, 2003.

[26] O. Chmaissem, H. Zheng, A. Huq, P. W. Stephens, and J. F. Mitchell, "Formation of $\mathrm{Co}^{3+}$ octahedra and tetrahedra in $\mathrm{YBaCo}_{4} \mathrm{O}_{8.1}$," Journal of Solid State Chemistry, vol. 181, no. 3, pp. 664-672, 2008.

[27] K. Amine, H. Tukamoto, H. Yasuda, and Y. Fujita, "Preparation and electrochemical investigation of $\mathrm{LiMn}_{2-x} \mathrm{Me}_{x} \mathrm{O}_{4}$ (Me: $\mathrm{Ni}$, $\mathrm{Fe}$, and $x=0.5,1)$ cathode materials for secondary lithium batteries," Journal of Power Sources, vol. 68, no. 2, pp. 604-608, 1997.

[28] K. Amine, H. Tukamoto, H. Yasuda, and Y. Fujita, "A new threevolt spinel $\mathrm{Li}_{1+x} \mathrm{Mn}_{1.5} \mathrm{Ni}_{0.5} \mathrm{O}_{4}$ for secondary lithium batteries," Journal of the Electrochemical Society, vol. 143, no. 5, pp. 16071613, 1996.

[29] H. Duncan, Y. Abu-Lebdeh, and I. J. Davidson, "Study of the cathode-electrolyte interface of $\mathrm{LiMn}_{1.5} \mathrm{Ni}_{0.5} \mathrm{O}_{4}$ synthesized by a sol-gel method for li-ion batteries," Journal of the Electrochemical Society, vol. 157, no. 4, pp. A528-A535, 2010.

[30] D. Liu, J. Han, and J. B. Goodenough, "Structure, morphology, and cathode performance of $\mathrm{Li}_{1-x}\left[\mathrm{Ni}_{0.5} \mathrm{Mn}_{1.5}\right] \mathrm{O}_{4}$ prepared by coprecipitation with oxalic acid," Journal of Power Sources, vol. 195, no. 9, pp. 2918-2923, 2010.

[31] R. Santhanam and B. Rambabu, "Research progress in high voltage spinel $\mathrm{LiNi}_{0.5} \mathrm{Mn}_{1.5} \mathrm{O}_{4}$ material," Journal of Power Sources, vol. 195, no. 17, pp. 5442-5451, 2010.

[32] M. Aklalouch, R. M. Rojas, J. M. Rojo, I. Saadoune, and J. M. Amarilla, "The role of particle size on the electrochemical properties at 25 and at $55^{\circ} \mathrm{C}$ of the $\mathrm{LiCr}_{0.2} \mathrm{Ni}_{0.4} \mathrm{Mn}_{1.4} \mathrm{O}_{4}$ spinel as $5 \mathrm{~V}$-cathode materials for lithium-ion batteries," Electrochimica Acta, vol. 54, no. 28, pp. 7542-7550, 2009.

[33] M. Aklalouch, J. M. Amarilla, R. M. Rojas, I. Saadoune, and J. M. Rojo, "Chromium doping as a new approach to improve the 
cycling performance at high temperature of $5 \mathrm{~V} \mathrm{LiNi}_{0.5} \mathrm{Mn}_{1.5} \mathrm{O}_{4}$ based positive electrode," Journal of Power Sources, vol. 185, no. 1, pp. 501-511, 2008.

[34] I. Soibam, S. Phanjoubam, H. N. K. Sarma, and C. Prakash, "Synthesis and characterization of ultra-fine zinc substituted lithium ferrites," in Proceedings of the AIP Conference, vol. 1003, pp. 136-138, 2008.

[35] I. Soibam, S. Phanjoubam, H. B. Sharma, and H. N. K. Sarma, "Synthesis of lithium zinc ferrite powders by citrate precursor gel formation method," Indian Journal of Physics, vol. 82, no. 5, pp. 611-614, 2008.

[36] M. Maisnam, S. Phanjoubam, H. N. K. Sarma, C. Prakash, L. Radhapiyari Devi, and O. P. Thakur, "Structural and DC resistivity behaviour of Li-Mn-Ni ferrites substituted with trace amount of $\mathrm{Co}^{2+}$, Physica B, vol. 370, no. 1-4, pp. 1-5, 2005.

[37] V. Marinova, "Optical properties of $\mathrm{Bi}_{12} \mathrm{TiO}_{20}$ doped with $\mathrm{Al}$, $\mathrm{P}, \mathrm{Ag}, \mathrm{Cu}, \mathrm{Co}$ and co-doped with $\mathrm{Al}+\mathrm{P}$ single crystals," Optical Materials, vol. 15, no. 2, pp. 149-158, 2000. 

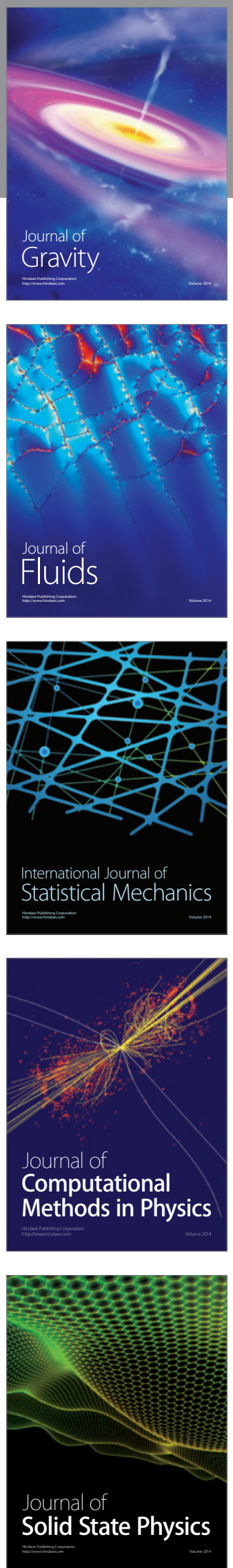

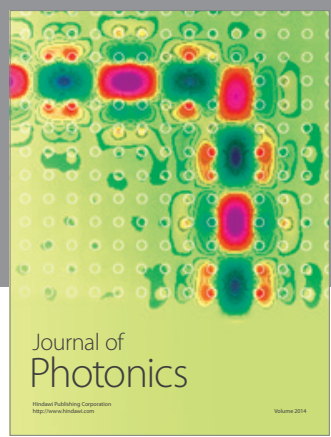

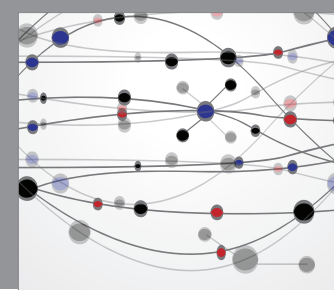

The Scientific World Journal

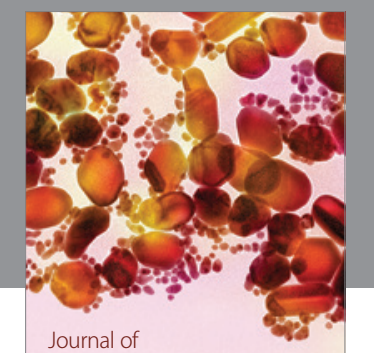

Soft Matter
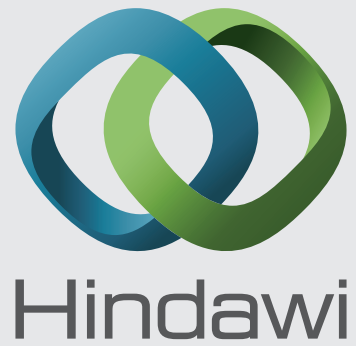

Submit your manuscripts at

http://www.hindawi.com
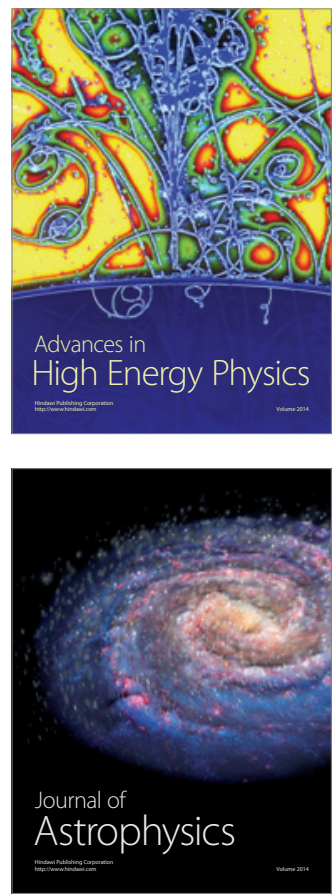
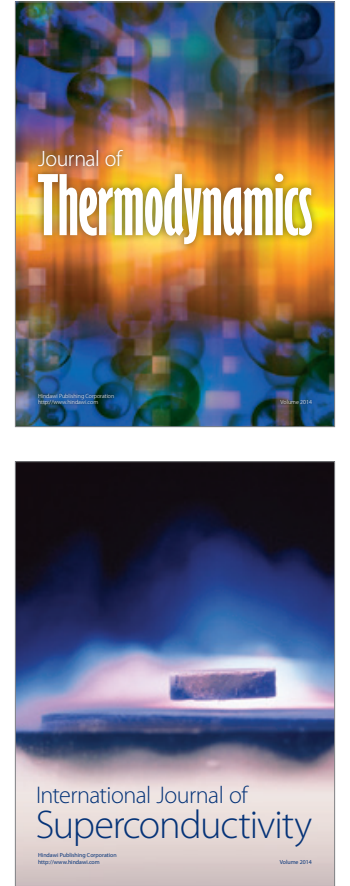
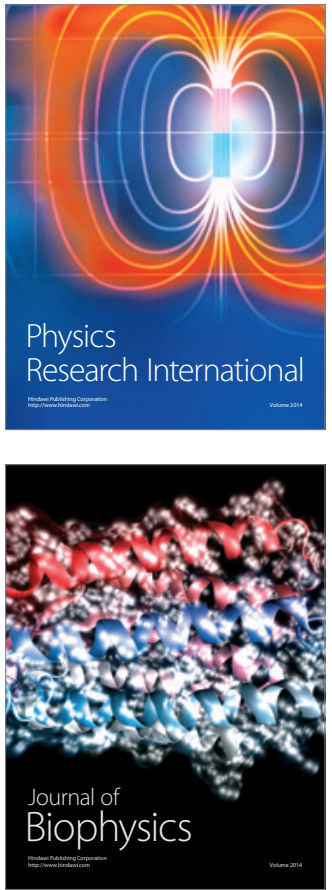
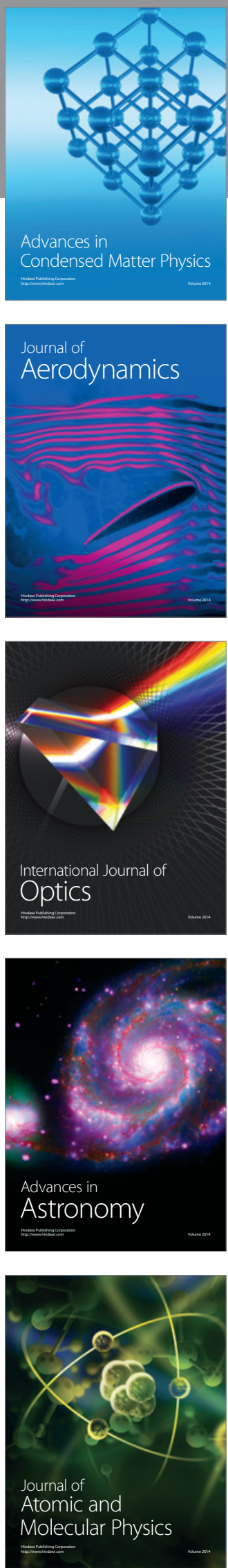